Perspectiva de Familia (Perspect. Fam.) ISSN 2415-5187 (impreso), ISSN 2519-1748 (en línea), año 2019, vol. 4, pp. 27-45

\title{
Influencia de la estructura y funcionalidad familiar en la resiliencia de adolescentes en situación de pobreza
}

\author{
Influence of family structure and functionality on the resilience \\ of adolescents living in poverty
}

\author{
Gabriela P. García Zavala \\ María L. Diez Canseco Briceño \\ Universidad Católica San Pablo, Perú
}

Correspondencia: gpgarcia@ucsp.edu.pe

Recibido: 05-06-2019. Revisado: 28-08-2019. Aceptado: 04-10-2019

Citarlo como: García, G., \& Diez Canseco, M. L. (2019). Influencia de la estructura y funcionalidad familiar en la resiliencia de adolescentes en situación de pobreza. Perspectiva de Familia, 4, pp. 27-45.

\section{Resumen}

La resiliencia en la adolescencia es muy importante, en especial en poblaciones que viven en pobreza. El papel de la familia en el desarrollo de esta es fundamental, convirtiéndose en factor protector; pero la pobreza puede afectarla modificando sus relaciones y convertirla en un factor de riesgo. Esta investigación pretendió identificar la influencia de la estructura y funcionalidad familiar en la resiliencia de adolescentes en situación de pobreza, por lo cual se realizó un estudio ex post facto retrospectivo simple. La muestra estuvo constituida por 393 estudiantes de entre 12 a 17 años provenientes de colegios de Arequipa que, según el Sistema de Focalización de Hogares (SISFOH), se encontraban en centros poblados en situación de pobreza y pobreza extrema. Se los evaluó con tres escalas: la de Resiliencia (ER), de Wagnild y Young (1993); la de Evaluación de la Adaptabilidad y Cohesión Familiar (Faces III), de Olson, Portner y Lavee (1985), y la de Comunicación Familiar (PACS), de Barnes y Olson (1982); además de una encuesta sobre estructura familiar. Se 
encontró que la estructura y funcionalidad familiar influyen en un $18.1 \%$ en la resiliencia de estos adolescentes; concluyendo que esta última es un proceso interaccional donde intervienen diversos factores, siendo la familia uno de los principales. Algunas características específicas de la familia, como el proceder de una familia rígida y desligada, reducen significativamente el desarrollo de la resiliencia, mientras que el ser mujer y el tener mayor edad la favorecen muy significativamente.

Palabras clave: Resiliencia, familia, funcionalidad, adolescencia, pobreza.

\begin{abstract}
Resilience in adolescence is very important, especially in populations living in poverty. The role of the family in the development of this is fundamental, becoming a protective factor; but poverty can affect family by modifying its relationships and turning it into a risk factor. This research aimed to identify the influence of family structure and functionality on the resilience of adolescents living in poverty; therefore, an ex post facto retrospective single study was carried out. The sample consisted of 393 students aged between 12 and 17 years old from Arequipa schools that according to SISFOH (Home Focusing System) were in populated centers in situations of poverty and extreme poverty. They were evaluated with three scales: Resilience Scale (RS), by Wagnild and Young (1993); Family Adaptability and Cohesion Evaluation Scale (Faces III), by Olson, Portner and Lavee (1985), and the Parent-Adolescent Communication Scale (PACS), by Barnes and Olson (1982); additionally a family structure survey. It was found that family structure and functionality influence $18.1 \%$ in the level of resiliency of these adolescents; concluding that the last is an interactional process where several factors are involved, being the family one of the most important. Some specific characteristics of the family, such as proceeding from a rigid and disengaged family, significantly reduce the development of resilience; meanwhile to be woman and older favor it very significantly.
\end{abstract}

Key words: Resilience, family, functionality, adolescence, poverty.

La población adolescente siempre ha sido considerada como un sector vulnerable, en especial porque en esta etapa de la vida es necesario buscar y conseguir la salud de la persona, pero no solo desde el punto de vista biológico, sino principalmente desde el ámbito psicosocial (Ministerio de Salud [MINSA], 2010a). En Perú, hasta el año 2015, esta población representaba el $18.6 \%$ de la población total (MINSA, 2017). En Arequipa hay más de doscientos mil adolescentes (Instituto Nacional de Estadística e Informática [INEI], 2013; MINSA, 2010a), siendo una de las ciudades con mayor población de esta edad. 
El factor pobreza es uno de los principales problemas que afecta a los adolescentes, ya que trae consigo consecuencias muy grandes que, a veces, no pueden ser manejadas por los miembros de este grupo (Kotliarenco, Cáceres \& Fontecilla, 1997). Esto lleva a que puedan manifestar comportamientos no adecuados en varios de los ámbitos en los que se desenvuelven (Vinaccia, Quiceno \& Moreno, 2007). Con el paso de los años, la pobreza se ha reducido en Perú; pero la población adolescente es una de las que presenta un mayor índice que el promedio nacional, agudizándose en zonas rurales (MINSA, 2017).

Como dijimos anteriormente, la pobreza afecta a este grupo en distintos ámbitos; uno de ellos es el de la educación, ya que del total de adolescentes que no asisten a la escuela, el $27.6 \%$ lo hace por falta de dinero, lo cual hace que tengan que trabajar (INEI, 2018). Otro dato que ejemplifica esta relación es que en Arequipa la asistencia a educación secundaria es del $88.3 \%$, cifra que varía dependiendo del nivel de pobreza de las personas. En el caso de la población que está considerada como «Pobre no extremo», únicamente asiste un $75.7 \%$, y en el caso de la población «Pobre extremo», un 60.2\% (Estadística de la Calidad Educativa, Ministerio de Educación [ESCALE-MINEDU], 2014). En Arequipa, solamente el $65.7 \%$ de colegios cuentan con los tres servicios básicos, es decir, agua, luz y desagüe (ESCALEMINEDU, 2014).

Esta es la realidad a la que diariamente se enfrentan los adolescentes que viven en situación de pobreza, y, ante ella, podrán responder de diversas maneras. Unos lograrán salir adelante y progresarán enfrentando las adversidades del ambiente, mientras que otros no podrán sobreponerse. Y esta actitud que presentan tiene mucha relación con la resiliencia, que se considera como aquella capacidad que permite que la persona pueda proyectarse a futuro a pesar de que en su vida se presenten condiciones poco adecuadas o adversas (Kotliarenco et al., 1997).

En el tema de resiliencia se deben considerar factores de riesgo y factores de protección. Los primeros se refieren a ciertas características o cualidades que facilitan que se produzca algún tipo de daño tanto a nivel físico como psicológico; los segundos se entienden como ciertas condiciones o medios que favorecen el crecimiento, pudiendo reducir los efectos que circunstancias desfavorables hayan producido en algunos casos (Munist et al., 1998). Un factor considerado altamente de riesgo es la pobreza, ya que las personas y sus familias están expuestas a condiciones precarias que atentan contra su salud física y mental (Kotliarenco et al., 1997).

En este sentido, la familia se presenta como un elemento fundamental, puesto que puede convertirse en un factor de riesgo o protector. Se considera como un factor de riesgo cuando se dan prácticas de crianza inconducentes a su desarrollo, así como 
abuso y conflictos familiares (Vinaccia et al., 2007). El Ministerio de Salud considera que entre los factores que causan mayor estrés en los adolescentes en el Perú están los problemas con los parientes, y estos se presentan con mayor magnitud en la sierra (MINSA, 2010b). Hay investigaciones que explican que existe una diferencia importante entre un adolescente resiliente de aquel que no lo es, la cual radica principalmente en el grado de exposición a la adversidad familiar a la que se han visto enfrentados en la niñez (Vinaccia et al., 2007).

En contraposición a esto, la familia puede ser un factor protector que promueva la resiliencia. Esto depende de su naturaleza y de los atributos que presenta, tales como cohesión, ternura y preocupación por los hijos (Guillén, 2005; Kotliarenco et al., 1997). Algunos autores consideran importante la presencia de una relación emocional estable, al menos con uno de los padres, como uno de los recursos más importantes en la resiliencia (Kotliarenco et al., 1997).

Por lo tanto, la familia se convierte en un componente muy importante en la resiliencia, ya que puede ser un factor protector o, al verse afectado por la pobreza que modifica sus relaciones, uno de riesgo. Por ende, se hace necesaria una investigación que considere diversas variables de la familia en la formación de la resiliencia, en especial en los adolescentes que viven en pobreza.

En Latinoamérica, se han realizado diversos estudios sobre resiliencia en poblaciones con adversidad económica y en situación de vulnerabilidad (Aguiar \& Acle-Tomasini, 2012; Barcelta \& Gómez-Maqueo, 2012; Jadue, Galindo \& Navarro, 2005) que manifiestan que la familia podría ser un factor de riesgo o protección, dependiendo de si se presenta como un agente estresor o como un apoyo a la adaptación de sus miembros.

Vinaccia et al. (2007) llegaron a la conclusión de que la pobreza, las situaciones de crianza inadecuadas, los conflictos familiares y la enfermedad mental de los padres generaban una situación mayor de riesgo en los niños y adolescentes. Hay autores que proponen que frente a la pobreza, la familia se verá muy afectada en su constitución y en el desarrollo de sus relaciones (Kotliarenco et al., 1997), pudiendo anular así su papel en la formación de la resiliencia.

Por eso, se considera necesario avanzar en la investigación de estas variables e indagar en el impacto de la familia sobre la resiliencia de estos adolescentes que viven en pobreza; por lo cual, surge la siguiente interrogante: ¿Cuánto influye la estructura y funcionalidad familiar en la resiliencia de adolescentes en situación de pobreza? 


\section{Funcionalidad Familiar}

La funcionalidad familiar es entendida como la capacidad del sistema para enfrentar y superar cada una de las etapas del ciclo vital y las crisis por las que atraviesa la familia (Rangel, Valerio, Patiño \& García, 2004). El Modelo Circumplejo, desarrollado por Olson, se basa en la hipótesis central de que los sistemas familiares balanceados tienden a ser más funcionales en comparación con los sistemas no balanceados. Dentro de este modelo, considera tres dimensiones: Cohesión, Flexibilidad y Comunicación familiar (Olson, 200o).

La cohesión familiar es entendida como el lazo emocional que la pareja y miembros de la familia tienen entre sí. Se centra en cómo el sistema logra tener su balance frente a la separación y a la unión (Olson \& Gorall, 2003). Existen cuatro niveles en esta dimensión que tratan de describir las diferentes formas en las que se manifiesta dentro de la familia, estos van desde muy bajo a muy alto, presentándose las familias en el siguiente orden: desligada, separada, conectada y aglutinada. Los niveles centrales son los que se consideran óptimos, en oposición a los extremos, que se consideran problemáticos (Sotil \& Quintana, 2002; Zambrano, 2011).

La adaptabilidad familiar se relaciona con la flexibilidad, refiriéndose al balance que logra la familia frente al cambio (Olson \& Gorall, 2003). Zambrano (2011) la define como esa habilidad de la familia para poder cambiar sus estructuras de poder, las relaciones basadas en roles y las reglas, en las situaciones causadas por el estrés situacional o aquellas que se presentan por el desarrollo. Se distinguen cuatro niveles que van desde muy bajo a muy alto, pudiendo ser la familia rígida, estructurada, flexible y caótica. Los estilos extremos serán los menos funcionales, mientras que los centrales serán los más óptimos (Sotil \& Quintana, 2002; Zambrano, 2011).

Olson y Gorall (2003) explican que la comunicación es la tercera dimensión del Modelo Circumplejo, y es considerada como una dimensión facilitadora. La comunicación familiar es fundamental, ya que logra preparar a las familias para que modifiquen sus niveles de cohesión y flexibilidad. Mediante el uso de herramientas positivas de comunicación, es posible que ellas puedan cambiar los niveles de cohesión y flexibilidad cuando se encuentran en etapas de desarrollo o situaciones demandantes.

\section{Resiliencia}

La palabra resiliencia proviene del latín «resilio, que significa volver atrás, volver de un salto, resaltar, rebotar» (Kotliarenco et al., 1997, p. 5). El término suele definirse de diferentes formas dependiendo del área de conocimiento en la cual se estudia. 
La definición más aceptada de resiliencia es la que la considera como una capacidad que presenta una persona o un grupo para salir adelante y proyectarse hacia el futuro a pesar de la adversidad y de las dificultades que se puedan presentar en su vida (Kotliarenco et al., 1997). Si bien existen varias investigaciones realizadas en distintas áreas sobre el tema, aún falta llegar a un consenso sobre el término. Diferentes son las posturas sobre sus características, si es un proceso o un atributo, si se construye o es innato, si es adaptación o superación de la adversidad, si se presenta constantemente o es solo circunstancial (Alarcón, 2009).

Wagnild y Young (1993), autoras de la Escala de Resiliencia, la consideran como la capacidad para afrontar con éxito el cambio o la desgracia; también la entienden como una «característica de la personalidad que modera el efecto negativo del estrés y fomenta la adaptación. Esta se ha utilizado para describir a personas que muestran valentía y adaptabilidad ante los infortunios de la vida» (p. 6).

Cabe resaltar la postura de la resiliencia desde el punto de vista interaccional, el cual pone énfasis en que esta se va formando a través de las interacciones que desarrolla la persona a lo largo de su vida (Saavedra \& Villalta, 2008).

Salgado (2005) explica que existen eventos, situaciones o realidades que pueden ser favorables o desfavorables para su desarrollo. De este análisis es que se derivan los factores protectores y los factores de riesgo.

Según Guillén (2005) y Salgado (2005), los factores protectores se pueden dividir en tres: personales, familiares y de apoyo social. Dentro de los factores protectores están algunas características personales como la autoestima, la autonomía, la orientación social, la empatía, entre otros. Respecto a los familiares, se presenta la cohesión, calidez, presencia de la madre, comunicación abierta, estructura familiar sin disfuncionalidades importantes y adecuadas pautas de crianza. En el apoyo social, estará el reconocimiento de su valor y personalidad en los distintos ámbitos en los que se desenvuelva.

Los factores de riesgo se consideran características o cualidades que están unidas a una alta probabilidad de dañar la salud tanto física como psicológica (Munist et al., 1998). Estos también pueden dividirse en tres grupos: individuales, interpersonales y ambientales. Dentro del primero se encuentran el pobre control de impulsos, la falta de motivación, la conducta criminal, entre otros (Aguiar \& Acle-Tomasini, 2012; Becoña, 2006); en el segundo, la inadecuada comunicación familiar, el conflicto familiar, el apego deficiente, los estilos de crianza inadecuados, el consumo de alcohol o drogas de parte de los padres, el bajo rendimiento escolar, el 
rechazo de pares y la asociación con grupos de compañeros antisociales (Aguiar \& Acle-Tomasini, 2012; Becoña, 2006); y en el tercero, la pobreza, privaciones económicas, desorganización vecinal e inseguridad en los barrios (Becoña, 2006).

\section{Pobreza}

Uno de los problemas más importantes a los que se enfrenta el Perú es la pobreza. No existe un consenso sobre el concepto, este se va formando dependiendo del tipo de pobreza que se considera; aunque, de manera general, «puede entenderse como la falta de capacidad para alcanzar y mantener un nivel de vida aceptable, por lo tanto, una persona pobre será aquella que sufra alguna privación que le impida el logro de este objetivo» (Otero, 2002, p. 11).

Hay distintas maneras de medir la pobreza, desde la medición de los hogares y su acceso a los servicios básicos hasta los métodos que la miden según el poder adquisitivo (Ministerio de Economía y Finanzas [MEF], 2009). La presente investigación identifica la pobreza según el Sistema de Focalización de Hogares (SISFOH), que es un instrumento que intenta responder a las necesidades de información social, por lo cual, describe las características socioeconómicas de los hogares (características de la vivienda, del hogar y de sus miembros). En este contexto, mediante la focalización, se asignan los subsidios del Estado a las familias más pobres y vulnerables. Este programa se encontraría clasificando a la pobreza según el método no monetario, midiéndola conforme a los indicadores de condiciones de vida de los hogares. Según Otero (2002), la pobreza puede dividirse en pobreza absoluta, relativa, coyuntural y estructural. Este estudio, al tomar en cuenta la pobreza según el SISFOH, considerará el enfoque estructural y absoluto, puesto que se consideran las privaciones que posee la familia actualmente, no necesariamente guiados por la coyuntura ni el contexto.

\section{Método}

\section{Diseño}

El diseño de esta investigación es el de un estudio ex post facto retrospectivo simple (Montero \& León, 2007), ya que el hecho se produjo con anterioridad, por lo que, como segundo paso, se busca analizar las posibles causas. No se intenta modificar el fenómeno o la situación objeto de análisis (en este caso, la resiliencia), las variables ya han ocurrido.

Montero y León (2005) proponen denominar a las variables dependiente e independiente, dado que, aunque no se experimentará con ellas ni se las modificará, se dará 
una relación explicativa. En este caso se inicia analizando la variable dependiente (resiliencia), seguidamente de las variables independientes (familia: estructura y funcionalidad) que puedan explicarla.

\section{Participantes}

Se utilizó un tipo de muestreo no probabilístico (Hernández, Fernández \& Baptista, 2006). Fueron evaluados 393 alumnos de dos colegios de Arequipa ubicados en centros poblados en situación de pobreza, según el SISFOH, que cursaban 2..o, 3.․, $4 .^{\circ}$ y $5 .{ }^{\circ}$ de secundaria. Participaron en total 199 hombres (50.6\%) y 194 mujeres $(49.4 \%)$.

\section{Instrumentos}

La Escala de Resiliencia de Wagnild y Young fue elaborada en 1988 y revisada por las mismas autoras en 1993. En el año 2002 fue adaptada a la población peruana por Novella. Está compuesta por 25 ítems, los cuales puntúan en una escala tipo Likert de 7 puntos, los puntajes más altos serán indicadores de mayor resiliencia, el rango de puntaje varía entre 25 y 175. Presenta una confiabilidad calculada por el método de consistencia interna con el coeficiente alfa de Cronbach de .89 (Salgado, 2009). En el año 2014, Castilla, Caycho, Shimabukuro, Valdivia y Torres-Calderón hicieron otro análisis psicométrico en el Perú, ellos encontraron que la escala presentaba una elevada consistencia interna con un alfa de Cronbach de .906. En un estudio realizado por Cabello, Sucacahua, Lazo, Najarro y Palomino (2016) se calculó la validez mediante un análisis factorial y se encontró que la escala presentaba una estructura unidimensional.

El FACES (Family Adaptability and Cohesion Evaluation Scales), desarrollado por Olson, Portner y Lavee (1985), consta de 20 ítems con una escala de respuesta de cinco puntos, y presenta una fiabilidad global de .86 según el alfa de Cronbach, para la escala de cohesión es de .86, y para la escala de adaptabilidad, de .71 (Jiménez, Musitu \& Murgui, 2006). Ha sido utilizada en diversas investigaciones en el Perú, y fue adaptada por Reusche consiguiendo una confiabilidad en la escala de cohesión de .83 y en la escala de adaptabilidad de .8o (Zambrano, 2011).

El Cuestionario de Comunicación Familiar (PACS), de Barnes y Olson (1982), se compone de dos escalas, la primera evalúa la comunicación entre los hijos y la madre, y la segunda evalúa la comunicación con el padre. Cada escala consta de 20 ítems con un sistema de respuesta tipo Likert de cinco puntos. Representa dos grandes dimensiones de la comunicación entre padres e hijos: la apertura en la comunicación y los problemas en la comunicación (Jiménez, Musitu \& Murgui, 
2006). Este cuestionario ha sido validado en el Perú presentando una adecuada consistencia interna calculada con el alfa de Cronbach, para la subescala de apertura, de .87 , y para la subescala de problemas en la comunicación tanto con el padre como con la madre, de .68 y de .66, respectivamente (Bueno, 1996).

Se aplicó una encuesta estructurada con el fin de evaluar la estructura familiar, en la cual se presentaron opciones para que el encuestado defina cómo está compuesta la familia con la que vive.

\section{Procedimiento}

Se ubicaron los centros poblados en Arequipa que, según el SISFOH, presentaban un mayor índice de pobreza y pobreza extrema. Se eligieron a dos colegios localizados en los centros poblados de referencia y se solicitó a los directores de los mismos el permiso para evaluar a los estudiantes.

Se evaluó de manera grupal a los estudiantes aplicando la Escala de Resiliencia, de Wagnild y Young (1993), la escala de Cohesión y Adaptabilidad familiar (FACES III), de Olson, Portner y Lavee (1985), el cuestionario de Comunicación Familiar (PACS), de Barnes y Olson (1982), y la encuesta de estructura familiar.

\section{Análisis de datos}

Para el procesamiento de los datos se utilizó el programa estadístico SPSS versión 20.0 (IBM Corp, 2011), inicialmente se realizó un análisis descriptivo de las variables y luego, para calcular la influencia de las variables familiares en la resiliencia, una regresión logística binaria. Para la ejecución de este análisis se diferenció la variable dependiente, que era la resiliencia, de las variables independientes, que fueron la estructura y la funcionalidad familiar.

\section{Resultados}

En primer lugar, se encontró que la mayoría de los evaluados son resilientes $(70.2 \%)$, siendo este porcentaje mayor en mujeres $(78.4 \%)$ que en hombres $(62.3 \%)$. 
Tabla 1.

Sexo y Resiliencia

\begin{tabular}{|c|c|c|c|c|c|}
\hline & & \multicolumn{3}{|c|}{ Resiliencia } & \multirow{2}{*}{ Total } \\
\hline & & No resiliente & & & \\
\hline \multirow{4}{*}{ Sexo } & \multirow{2}{*}{ Mujer } & $\mathrm{F}$ & 42 & 152 & 194 \\
\hline & & $\%$ & $21.6 \%$ & $78.4 \%$ & $100.0 \%$ \\
\hline & \multirow{2}{*}{ Varón } & $\mathrm{F}$ & 75 & 124 & 199 \\
\hline & & $\%$ & $37.7 \%$ & $62.3 \%$ & $100.0 \%$ \\
\hline \multirow[t]{2}{*}{ Total } & & $\mathrm{F}$ & 117 & 276 & 393 \\
\hline & $\%$ & $\%$ & $29.8 \%$ & $70.2 \%$ & $100.0 \%$ \\
\hline
\end{tabular}

En cuanto a la resiliencia según la estructura familiar, se observa que los estudiantes que provienen de familias extendidas y nucleares más del $70 \%$ son resilientes; mientras que los que provienen de hogares donde los padres no se hallan presentes menos de la mitad son resilientes $(42.1 \%)$.

\section{Tabla 2.}

\section{Resiliencia y Estructura Familiar}

\begin{tabular}{|c|c|c|c|c|c|}
\hline & & & \multicolumn{2}{|c|}{ Resiliencia } & \multirow{2}{*}{ Total } \\
\hline & & & No resiliente & Resiliente & \\
\hline \multirow{10}{*}{ Familia } & \multirow{2}{*}{ Ausencia de padres } & $\mathrm{F}$ & 11 & 8 & 19 \\
\hline & & $\%$ & $57 \cdot 9 \%$ & $42.1 \%$ & $100.0 \%$ \\
\hline & \multirow{2}{*}{ Monoparental } & $\mathrm{F}$ & 16 & 35 & 51 \\
\hline & & $\%$ & $31.4 \%$ & $68.6 \%$ & $100.0 \%$ \\
\hline & \multirow{2}{*}{ Reconstruida } & $\mathrm{F}$ & 10 & 22 & 32 \\
\hline & & $\%$ & $31.3 \%$ & $68.8 \%$ & $100.0 \%$ \\
\hline & \multirow{2}{*}{ Extendida } & $\mathrm{F}$ & 10 & 31 & 41 \\
\hline & & $\%$ & $24.4 \%$ & $75.6 \%$ & $100.0 \%$ \\
\hline & \multirow{2}{*}{ Nuclear } & $\mathrm{F}$ & 70 & 180 & 250 \\
\hline & & $\%$ & $28.0 \%$ & $72.0 \%$ & $100.0 \%$ \\
\hline \multirow[t]{2}{*}{ Total } & & $\mathrm{F}$ & 117 & 276 & 393 \\
\hline & $\%$ & $29.8 \%$ & $70.2 \%$ & $100.0 \%$ & $100.0 \%$ \\
\hline
\end{tabular}

En cuanto a la cohesión familiar, se observa que hay un mayor porcentaje de resilientes en los evaluados que provienen de familias aglutinadas (91.4\%) y conectadas (80.4\%); en contraste con los que provienen de familias desligadas, siendo resilientes solamente un $53.4 \%$. 
Tabla 3.

Resiliencia y Cohesión Familiar

\begin{tabular}{|c|c|c|c|c|c|}
\hline & & & \multicolumn{2}{|c|}{ Resiliencia } & \multirow{2}{*}{ Total } \\
\hline & & & No resiliente & Resiliente & \\
\hline \multirow{8}{*}{ Cohesión } & \multirow{2}{*}{ Desligada } & $\mathrm{F}$ & 54 & 62 & 116 \\
\hline & & $\%$ & $46.6 \%$ & $53.4 \%$ & $100.0 \%$ \\
\hline & \multirow{2}{*}{ Separada } & $\mathrm{F}$ & 38 & 92 & 130 \\
\hline & & $\%$ & $29.2 \%$ & $70.8 \%$ & $100.0 \%$ \\
\hline & \multirow{2}{*}{ Conectada } & $\mathrm{F}$ & 22 & 90 & 112 \\
\hline & & $\%$ & $19.6 \%$ & $80.4 \%$ & $100.0 \%$ \\
\hline & \multirow{2}{*}{ Aglutinada } & $\mathrm{F}$ & 3 & 32 & 35 \\
\hline & & $\%$ & $8.6 \%$ & $91.4 \%$ & $100.0 \%$ \\
\hline \multirow[t]{2}{*}{ Total } & & $\mathrm{F}$ & $\mathrm{n}$ & 276 & 393 \\
\hline & $\%$ & $29.8 \%$ & $70.2 \%$ & $100.0 \%$ & $100.0 \%$ \\
\hline
\end{tabular}

Respecto a la adaptabilidad familiar, se encontró que solamente el $14.3 \%$ de los participantes que pertenecen a una familia rígida eran resilientes; mientras que, si su familia era estructurada, flexible o caótica, el porcentaje de resilientes superaba el $69 \%$.

Tabla 4 .

Resiliencia y Adaptabilidad Familiar

\begin{tabular}{|c|c|c|c|c|c|}
\hline & & & \multicolumn{2}{|c|}{ Resiliencia } & \multirow{2}{*}{ Total } \\
\hline & & & No resiliente & Resiliente & \\
\hline \multirow{8}{*}{ Adaptabilidad } & \multirow{2}{*}{ Rígida } & $\mathrm{F}$ & 6 & 1 & 7 \\
\hline & & $\%$ & $85.7 \%$ & $14.3 \%$ & $100.0 \%$ \\
\hline & \multirow{2}{*}{ Estructurada } & $\mathrm{F}$ & 15 & 34 & 49 \\
\hline & & $\%$ & $30.6 \%$ & $69.4 \%$ & $100.0 \%$ \\
\hline & \multirow{2}{*}{ Flexible } & $\mathrm{F}$ & 38 & 85 & 123 \\
\hline & & $\%$ & $30.9 \%$ & $69.1 \%$ & $100.0 \%$ \\
\hline & \multirow{2}{*}{ Caótica } & $F$ & 58 & 156 & 214 \\
\hline & & $\%$ & $27.1 \%$ & $72.9 \%$ & $100.0 \%$ \\
\hline \multirow[t]{2}{*}{ Total } & & $\mathrm{F}$ & 117 & 276 & 393 \\
\hline & $\%$ & $29.8 \%$ & $70.2 \%$ & $100.0 \%$ & $100.0 \%$ \\
\hline
\end{tabular}

Según la comunicación familiar, el porcentaje más alto de alumnos resilientes $(78.7 \%)$ se encuentra en el grupo que presenta una buena comunicación, seguido del grupo que presenta una comunicación regular (69.2\%) y mala (64.1\%). 


\section{Tabla 5.}

\section{Resiliencia y Comunicación Familiar}

\begin{tabular}{|c|c|c|c|c|c|}
\hline & & & \multicolumn{2}{|c|}{ Resiliencia } & \multirow{2}{*}{ Total } \\
\hline & & & No resiliente & Resiliente & \\
\hline \multirow{6}{*}{ 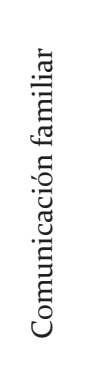 } & \multirow{2}{*}{ Mala } & $\mathrm{F}$ & 37 & 66 & 103 \\
\hline & & $\%$ & $35.9 \%$ & $64.1 \%$ & $100.0 \%$ \\
\hline & \multirow{2}{*}{ Regular } & $\mathrm{F}$ & 61 & 137 & 198 \\
\hline & & $\%$ & $30.8 \%$ & $69.2 \%$ & $100.0 \%$ \\
\hline & \multirow{2}{*}{ Buena } & $\mathrm{F}$ & 19 & 70 & 89 \\
\hline & & $\%$ & $21.3 \%$ & $78.7 \%$ & $100.0 \%$ \\
\hline \multirow[t]{2}{*}{ Total } & & $\mathrm{F}$ & 117 & 273 & 390 \\
\hline & $\%$ & $30.0 \%$ & $70.0 \%$ & $100.0 \%$ & $100.0 \%$ \\
\hline
\end{tabular}

El modelo de regresión logística binaria aplicado (ver Tabla 6) puede explicar el $18.1 \%$ de la resiliencia en los adolescentes en situación de pobreza.

En cuanto a las variables familiares que influyen en la resiliencia, se ha encontrado que, considerando la adaptabilidad, el provenir de una familia rígida disminuye significativamente en un $93 \%$ las probabilidades de ser resiliente, en contraste de si el adolescente proviene de una familia flexible. En cuanto a la cohesión familiar, se encontró que los adolescentes que provienen de familias desligadas tienen $80 \%$ menos de posibilidades de ser resilientes, en contraste con los que provienen de familias conectadas. Para el caso de la estructura familiar, provenir de un hogar donde los padres están ausentes reduce significativamente en un $83 \%$ las probabilidades de ser resiliente, lo cual no sucedería en una familia nuclear. En cuanto a la comunicación familiar, no se encontraron diferencias significativas.

Además de las variables de familia, se controlaron algunas variables demográficas como el sexo y la edad, las cuales dieron resultados altamente significativos. En lo que refiere al sexo, encontramos que ser varón reduce las posibilidades de ser resiliente en un $67 \%$. Sobre la edad, se encontró que, en los adolescentes, las posibilidades de ser resiliente aumentan en 1.4 veces conforme se hacen mayores. 
Tabla 6.

Análisis de regresión logística binaria

\begin{tabular}{|c|c|c|c|c|}
\hline & B & Sig. & $\operatorname{Exp}(B)$ & $\begin{array}{c}(1-\operatorname{Exp}(B)) \\
*_{100}\end{array}$ \\
\hline \multicolumn{5}{|l|}{ ADAPTABILIDAD } \\
\hline Flexible (variable de referencia) & & & 1 & \\
\hline Rígida & -2.642 & .023 & .071 & 92.9 \\
\hline Estructurada & .109 & .791 & 1.116 & \\
\hline Caótica & -.181 & .547 & .834 & \\
\hline \multicolumn{5}{|l|}{ COHESIÓN } \\
\hline Conectada (variable de referencia) & & & 1 & \\
\hline Desligada & -1.600 & .000 & .202 & 79.8 \\
\hline Separada & -.612 & .069 & .542 & \\
\hline Aglutinada & .862 & .196 & 2.367 & \\
\hline \multicolumn{5}{|l|}{ COMUNICACIÓN FAMILIAR } \\
\hline Mala (variable de referencia) & & & 1 & \\
\hline Regular & -.046 & .880 & .955 & \\
\hline Buena & .347 & .390 & 1.415 & \\
\hline \multicolumn{5}{|l|}{ ESTRUCTURA FAMILIAR } \\
\hline Nuclear (variable de referencia) & & & 1 & \\
\hline Ausencia de padres & -1.767 & .004 & .171 & 82.9 \\
\hline Monoparental & -.039 & .917 & .961 & \\
\hline Reconstruida & .451 & .332 & 1.570 & \\
\hline Extendida & .338 & .429 & 1.402 & \\
\hline \multicolumn{5}{|l|}{ SEXO } \\
\hline Mujer & & & 1 & \\
\hline Varón & -1.102 & .000 & .332 & 66.8 \\
\hline Edad (12-17 años) & .353 & .001 & 1.424 & - \\
\hline Constante & -2.182 & .181 & .113 & - \\
\hline
\end{tabular}

Nota: $n=390 ;$ Pseudo $R^{2}=0.1814$ 


\section{Discusión}

El objetivo de la investigación fue identificar la influencia que tenía la familia en la resiliencia de adolescentes que vivían en pobreza. Se encontró como resultado que la estructura y funcionalidad de la familia puede predecir en un $18.1 \%$ la resiliencia. Este dato obtenido presenta tres puntos particulares a discutir. Primero, la importancia de la familia aún en estas circunstancias de pobreza. Kotliarenco et al. (1997) explican que hay autores que consideran a la pobreza como una condición tan fuerte que atenta contra la misma familia, pudiendo afectar su estabilidad y el desarrollo adecuado de sus relaciones, y esto llevaría a que deje de ser ese factor protector. En la población estudiada, se ve que la familia en algunos casos cumple esa tarea de protección. Este resultado coincide con lo encontrado por Jadue et al. (2005).

Segundo, este dato respalda la teoría que considera a la resiliencia desde el punto de vista constructivista o interaccional (Saavedra \& Villalta, 2008), ya que la familia no podría ser el único factor que influya, sino que la resiliencia también se ve afectada por características personales y ambientales (Guillén, 2005). Se ha logrado medir el impacto de la familia, aunque solamente considerando sus características de estructura y funcionalidad, y se ha podido evidenciar que el peso que tiene es considerable.

Tercero, este resultado también demuestra una realidad importante de la persona, puesto que, al ser libre, no se puede ver determinada por ningún factor; pero, por ser social, tampoco se puede negar la influencia del ambiente, en este caso particular, el de la familia.

A continuación se hablará de las características particulares de la familia que favorecen a la resiliencia y que fueron encontradas en este estudio. La mayoría de los adolescentes provienen de familias nucleares $(63.6 \%)$, seguido de familias monoparentales (13.0\%), porcentajes muy similares a los obtenidos en otras investigaciones realizadas en la ciudad (Rivera \& Cahuana, 2016).

La presencia de los padres en la familia es muy importante, dado que el provenir de un hogar donde ellos están ausentes va a reducir significativamente las probabilidades de ser resiliente, en comparación con el hecho de provenir de una familia nuclear. Se puede entender este dato en vista de que, como Vinaccia et al. (2007) explican, uno de los recursos más importantes con los que cuentan las personas resilientes es el tener una relación emocional estable al menos con uno de sus padres, y en ese caso los adolescentes carecerían de esa figura. 
Los adolescentes que provienen de familias desligadas tienen $80 \%$ menos de posibilidades de ser resilientes, en contraste con aquellos que provienen de familias conectadas. Es decir que, cuando en una familia existe poca unión familiar - se pasa mucho tiempo separados tanto física como emocionalmente, las actividades y amistades son individuales y no familiares (Zambrano, 2011) -, ella no será ese punto de apoyo necesario para el desarrollo de la resiliencia. Por el contrario, si es una familia conectada - compartiendo mucho tiempo, espacio, amigos y actividades en familia (Zambrano, 2011)—, se permitirá el desarrollo de la resiliencia. Este dato está acorde a lo que Guillén (2005) proponía respecto a la importancia de la cohesión familiar para el desarrollo de la resiliencia.

El provenir de una familia rígida disminuye significativamente en un $93 \%$ las probabilidades de ser resiliente, en contraste de que se provenga de una familia flexible. Aunque no se hable mucho específicamente de la adaptabilidad de la familia para el desarrollo de la resiliencia, se la considera dentro de la funcionalidad familiar; además, tiene un desarrollo importante como característica personal (Aguiar \& Acle-Tomasini, 2012; De Oliveira, Lima, Silva \& Bueno, 2008; Guillén, 2005).

Por lo tanto, cuando una familia presente las características de flexibilidad, tales como permitir cambios, llegar a acuerdos en las decisiones, mantener una disciplina democrática, compartir e intercambiar roles (Zambrano, 2011), permitirá que el adolescente sea más flexible, se adapte rápidamente y posea esta característica como propia. Por el contrario, una familia que mantenga un liderazgo autoritario y un fuerte control parental, que se impongan decisiones o que la disciplina sea completamente estricta o rígida y su aplicación sea severa (Zambrano, 2011), definitivamente al adolescente le será muy difícil ser flexible. Y no hay que olvidar que un factor de riesgo es el sufrir castigos o violencia física por parte de los padres (Vinaccia et al., 2007).

Además de las variables de familia, se controlaron algunas variables demográficas como el sexo y la edad, que brindaron resultados altamente significativos. Analizando la variable sexo, se encontró que ser varón reduce las posibilidades de ser resiliente, en comparación con el hecho de ser mujer. Este dato no coincide con lo encontrado por Fergusson y Lynskey (1996), quienes hallaron que el hecho de ser mujer no hacía que uno sea más resiliente. Sin embargo, González-Arratia y Valdez (2013) encontraron que existía una diferencia significativa a favor de las mujeres en cuanto a la resiliencia.

Sobre la edad, se halló que, en los adolescentes, las posibilidades de resiliencia aumentan en 1.4 veces conforme se hacen mayores. Este dato se corresponde con la investigación realizada por Becoña, Míguez, López, Vázquez y Lorenzo (2006), 
aunque hay algunos estudios en los que no se encontró diferencia, como en el de Morales y Díaz (2011). Se considera que ese aumento encontrado se debe a que, mientras va avanzando la edad, la persona empieza a madurar; además es capaz de poder enfrentar los nuevos retos que se le van presentando y, por lo tanto, poner en práctica su capacidad resiliente.

\section{Referencias}

Aguiar, E., \& Acle-Tomasini, G. (2012). Resiliencia, factores de riesgo y protección en adolescentes mayas de Yucatán: Elementos para favorecer la adaptación escolar. Acta Colombiana de Psicología, 15(2), 53-64.

Alarcón, R. (2009). Psicología de la Felicidad. Lima: Editorial Universitaria.

Barcelta, B., \& Gómez-Maqueo, E. (2012). Fuentes de estrés y su influencia en la adaptación psicológica en jóvenes con adversidad económica. Revista En-Claves del Pensamiento, 6(12), 31.

Barnes, H. L., \& Olson, D. H. (1982). Parent-Adolescent Communication. En Olson, D. H., McCubbin, H. I., Barnes, H. L., Larsen, A., Muxen M., \& Wilson, M. (Eds.). Family inventories: Inventories used in a national survey of families across the family life cycle, (pp. 43-49). St. Paul, MN: University of Minnesota.

Becoña, E. (2006). Resiliencia: Definición, características y utilidad del concepto. Revista de Psicopatología y Psicología Clínica, 11(3), 125-146.

Becoña, E., Míguez, M., López, A., Vázquez, M., \& Lorenzo, M. (2006). Resiliencia y consumo de alcohol en jóvenes. Salud y drogas, 6(1), 89-111.

Bueno, R. (1996). Validación, confiabilidad y correlación entre las escalas de comunicación padres-adolescentes y satisfacción familiar en estudiantes de una universidad de Lima (tesis de maestría). Universidad San Martín de Porres, Lima, Perú.

Cabello, H. C., Sucacahua, J. C., Lazo, A. B., Najarro, M. M., \& Palomino, M. B. (2016). Validez y confiabilidad de la Escala de Resiliencia (Scale Resilience) en una muestra de estudiantes y adultos de la Ciudad de Lima. Revista Peruana de Psicología y Trabajo Social, 5(1), 121-136.

Castilla, H., Caycho, T., Shimabukuro, M., Valdivia, A., \& Torres-Calderón, M. (2014). Análisis exploratorio de la escala de resiliencia de Wagnild y Young en una muestra de adolescentes, jóvenes y adultos universitarios de Lima Metropolitana. Revista de Psicología de Arequipa, 4(1), 80-93.

Estadística de la Calidad Educativa-Ministerio de Educación. (2014). Estadística de la Calidad Educativa del año 2014 [base de datos en línea]. Recuperado el 30 de julio de 2015 de: http://escale.minedu.gob.pe/inicio 
Fergusson, D. M., \& Lynskey, M. T. (1996). Adolescent Resiliency to Family Adversity. Journal of Child Psychology and Psychiatry, 37(3), 281-292. doi:10.1111/j.1469-7610.1996.tbo1405.X

González-Arratia, N. I., \& Valdez, J. L. (2013). Resiliencia: Diferencia por Edad en Hombres y Mujeres Mexicanos. Acta de Investigación en Psicología, 3(1), 941955 .

Guillén, R. (2005). Resiliencia en la adolescencia. Revista de la Sociedad Boliviana de Pediatría, 44(1), 41-43.

Hernández, R., Fernández, C., \& Baptista, P. (2006). Metodología de la investigación (vol. 4). México: McGraw Hill.

IBM Corp (2011). IBM SPSS Statistics for Windows, Version 20.o. Armonk, NY: IBM Corp.

Instituto Nacional de Estadística e Informática. (2013). 11 de julio: Día mundial de la población. Recuperado el 30 de abril de 2015 de: http://www.inei.gob.pe/ media/MenuRecursivo/publicaciones_digitales/Est/Lib1157/libro.pdf

Instituto Nacional de Estadística e Informática. (2018). Estado de la Niñez y Adolescencia. Trimestre: Abril-Mayo-Junio 2018. Informe técnico N. 3-Setiembre 2018. Recuperado el 30 de abril de 2019 de: https://lampadia.com/assets/ uploads_documentos/9e829-03-informe-tecnico-no3_ninez-y-adolescencia-abr-may-jun2018.pdf

Jadue, G., Galindo, A., \& Navarro, L. (2005). Factores protectores y factores de riesgo para el desarrollo de la resiliencia encontrados en una comunidad educativa en riesgo social. Estudios pedagógicos, 31(2), 43-55.

Jiménez, T., Musitu, G., \& Murgui, S. (2006). Funcionamiento y comunicación familiar y consumo de sustancias en la adolescencia: El rol mediador del apoyo social. Revista de Psicología Social, 21(1), 21-34.

Kotliarenco, M., Cáceres, I., \& Fontecilla, M. (1997). Estado de arte en Resiliencia. Organización Panamericana de la Salud. Oficina Sanitaria Panamericana, Oficina Regional de la Organización Mundial de la Salud.

Ministerio de Economía y Finanzas. (2009). Mapa de pobreza: Métodos para medir pobreza. Recuperado el 20 de mayo de 2015 de: http://www.mef.gob.pe/index. php?option=com_content\&view=article\&id=370\&Itemid=100412\&lang=es

Ministerio de Salud. (2010a). Boletín informativo: «Etapa de Vida Adolescente n.o 1». Recuperado el 30 de abril de 2015 de: ftp://ftp2.minsa.gob.pe/descargas/ dgsp/MAIS/adolecente/boletino1-2010.pdf

Ministerio de Salud. (2010b). Boletín informativo: «Etapa de Vida Adolescente n.o 3». Recuperado el 30 de abril de 2015 de: ftp://ftp2.minsa.gob.pe/descargas/ dgsp/MAIS/adolecente/boletino3-2010.pdf 
Ministerio de Salud. (2017). Documento técnico: Situación de salud de los adolescentes y jóvenes en el Perú. Recuperado el 30 de abril de 2019 de: http://bvs. minsa.gob.pe/local/MINSA/4143.pdf

Montero, I., \& León, O. (2005). Sistema de clasificación del método en los informes de investigación en Psicología. International Journal of Clinical and Health Psychology, 5, 115-127.

Montero, I., \& León, O. (2007). A guide for naming research studies in Psychology. International Journal of Clinical and Health Psychology, 7(3), 847-862.

Morales, M., \& Díaz, D. (2011). Estudio comparativo de la resiliencia en adolescentes: El papel del género, la escolaridad y procedencia. Uaricha Revista de Psicología (Nueva época), 8(17), 62-77.

Munist, M., Santos, H., Kotliarenco, M., Suarez, E., Infante, F., \& Grotberg, E. (1998). El concepto de resiliencia. Manual de identificación y promoción de la resiliencia en niños y adolescentes, 7-17. Organización Panamericana de la Salud. Organización Mundial de la Salud.

Novella, A. (2002). Incremento de la resiliencia luego de la aplicación de un programa de psicoterapia breve en madres adolescentes (tesis de maestría). Universidad Nacional Mayor de San Marcos, Lima, Perú.

Oliveira, M. de, Lima, V., Silva, L., \& Bueno, C. (2008). Resiliencia: Análisis de las publicaciones en el periodo de 2000 a 2006. Psicología, ciencia e profissão, 28(4), 754-767.

Olson, D. (2000). Circumplex Model of Marital and Family Systems. Journal of Family Therapy, 22, 144-167.

Olson, D., \& Gorall, D. (2003). Circumplex model of marital and family systems. En Walsh, F. (Ed.). Normal Famlily Prcesses (3. ․ ed., pp. 514-547). New York: Guilford.

Olson, D., Portner, J., \& Lavee, Y. (1985). Manual de la Escala de Cohesión y Adaptabilidad Familiar (FACES III Manual). Minneapolis: Life Innovation.

Otero, G. (2002). Pobreza: Definición, determinantes y programas para su erradicación. Cuadernos de economía N.o 65. Ministerio de Economía de la provincia de Buenos Aires, 11-32.

Rangel, J., Valerio, L., Patiño, J., \& García, M. (2004). Funcionalidad Familiar en la adolescente embarazada. Revista de la Facultad de Medicina de la UNAM, 47(1), 24-27.

Rivera, R., \& Cahuana, M. (2016). Influencia de la familia sobre las conductas antisociales en adolescentes de Arequipa-Perú. Actualidades en Psicología, 30(120), 85-97. 
Saavedra, E., \& Villalta, M. (2008). Medición de las características resilientes, un estudio comparativo en personas entre 15 y 65 años. LIBERABIT, 14, 31-40.

Salgado, A. (2005). Inventario de Resiliencia para niños: Fundamentación teórica y construcción. Lima: USMP.

Salgado, A. (2009). Métodos e instrumentos para medir la resiliencia: Una alternativa peruana. LIBERABIT, 11, 41-48.

Sotil, A., \& Quintana, A. (2002). Influencias del clima familiar: Estrategias de aprendizaje e inteligencia emocional en el rendimiento académico. Revista de Investigación en Psicología, 5(1), 53-69.

Vinaccia, S., Quiceno, J., \& Moreno, E. (2007). Resiliencia en adolescentes. Revista Colombiana de Psicología, 16, 139-146.

Wagnild, G., \& Young, H. (1993). Escala de resiliencia (ER) [en línea]. Recuperado el 30 de julio de 2015 de: http://es.slideshare.net/ipsiasinstitutopsiquiatrico/39013071-escaladeresilienciadewagnildyyoung

Zambrano, A. (2011). Cohesión, adaptabilidad familiar y rendimiento académico en comunicación de alumnos de una Institución Educativa del Callao (tesis de maestría). Universidad San Ignacio de Loyola, Lima, Perú. 